\title{
Intelligent Cognitive Radio Models for Enhancing Future Radio Astronomy Observations
}

\author{
Ayodele Abiola Periola and Olabisi Emmanuel Falowo \\ Communication Research Group, Department of Electrical Engineering, University of Cape Town, Rondebosch, \\ Cape Town, South Africa \\ Correspondence should be addressed to Ayodele Abiola Periola; periola@crg.ee.uct.ac.za
}

Received 3 May 2016; Revised 12 August 2016; Accepted 14 September 2016

Academic Editor: Alberto J. Castro-Tirado

Copyright ( 2016 A. A. Periola and O. E. Falowo. This is an open access article distributed under the Creative Commons Attribution License, which permits unrestricted use, distribution, and reproduction in any medium, provided the original work is properly cited.

\begin{abstract}
Radio astronomy organisations desire to optimise the terrestrial radio astronomy observations by mitigating against interference and enhancing angular resolution. Ground telescopes (GTs) experience interference from intersatellite links (ISLs). Astronomy source radio signals received by GTs are analysed at the high performance computing (HPC) infrastructure. Furthermore, observation limitation conditions prevent GTs from conducting radio astronomy observations all the time, thereby causing low HPC utilisation. This paper proposes mechanisms that protect GTs from ISL interference without permanent prevention of ISL data transmission and enhance angular resolution. The ISL transmits data by taking advantage of similarities in the sequence of observed astronomy sources to increase ISL connection duration. In addition, the paper proposes a mechanism that enhances angular resolution by using reconfigurable earth stations. Furthermore, the paper presents the opportunistic computing scheme (OCS) to enhance HPC utilisation. OCS enables the underutilised HPC to be used to train learning algorithms of a cognitive base station. The performances of the three mechanisms are evaluated. Simulations show that the proposed mechanisms protect GTs from ISL interference, enhance angular resolution, and improve HPC utilisation.
\end{abstract}

\section{Introduction}

Astronomy is the scientific study of the universe by analysing astronomy source signals. Astronomy source signals can be received by either ground or space telescopes. Astronomy observations can also be categorised based on the signal source. Astronomy source signals that are observed by ground telescopes (GTs) can arise from optical, radio, and gravitational waves. In addition, astronomical source signals from X-ray, infra-red, and ultraviolet radiation are observed from space telescopes.

Radio and gravitational astronomy observations are complementary [1-3] and help in understanding the universe. The spectrum access of terrestrial radio astronomy observations is influenced by wavelength variation due to red shift and blue shift. Wavelength variation necessitates that GTs should have access to significant bandwidth resources. Terrestrial radio astronomy observations experience interference from the radio waves radiated by intersatellite links (ISLs) of low earth orbiting satellites. The use of ISLs is projected to increase due to small satellite proliferation [4-7].

Hence, a solution that protects terrestrial radio astronomy observations from interfering ISLs is needed. Such a solution can be designed using the cognitive radio (CR). The CR differentiates users based on their priority to access the radio spectrum. The two types of users recognised by a CR are primary users and secondary users with higher and lower spectrum access priority, respectively. The CR can be used to design interference protection schemes by using the interweaving or underlay spectrum sharing model. The interweaving spectrum sharing model enables secondary users to share access to the radio spectrum with primary users. The sharing is realised by informing secondary users of the spectrum access epochs of primary users. Being aware of primary user spectrum access epochs, the secondary users can use the spectrum when primary users are absent.

A CR based interweaving spectrum sharing framework that protects GTs from ISL interference is proposed in [8]. 
In [8], the primary user is the GT, while the secondary user is the ISL. The information on the epochs of astronomy source observation is accessible to the satellite and is used to determine the ISL activation epoch and duration. The CR is used to deactivate the ISL for a given duration, while terrestrial radio astronomy observations are ongoing. The results in [8] require further investigation to examine relations between GT interference protection and the similarities in the patterns of the observed astronomy sources.

Furthermore, organisations desiring to conduct radio astronomy observations can be classified based on GT availability. Some organisations can afford to construct their own GTs, while others convert unused earth stations to GTs. The conversion of unused earth stations is feasible due to the increasing use of fibre optic cables instead of satellites for broadband Internet access [9-14]. The discussion in [9-14] focuses on utilising converted unused satellite earth stations as GTs but does not consider the presence of terrestrial wireless networks in the destination electromagnetic environment of converted earth stations. In addition, the use of converted telescopes should also enhance the angular resolution of the terrestrial radio astronomy observations. Hoare and Rawlings [10] propose the use of a multimode telescope for satellite communications and terrestrial radio astronomy observations. The dynamic use of a multimode GT should enhance the angular resolution. The reconfigurable $\mathrm{CR}$ can be used to enhance the angular resolution when multimode GTs are used in terrestrial radio astronomy. The GT realised via conversion is also susceptible to interference when its destination environment comprises terrestrial wireless networks. Therefore, GTs require interference protection mechanisms.

In addition, terrestrial radio astronomy organisations also seek to maximise high performance computing (HPC) infrastructure utilisation. According to Barbosa et al. [15], the Atacama Large Millimetre/Submillimetre array (ALMA)'s HPC is underutilised due to observation limitation conditions. HPC utilisation can be improved by using techniques such as time multiplexing. However, other multiplex techniques such as duty cycle division multiplex which outperform time multiplexing have been proposed [16-18]. Therefore, a duty cycle multiplex scheme that can enhance HPC utilisation is required.

This paper addresses two goals for terrestrial radio astronomy organisations using converted GTs. It proposes mechanisms that optimise the conduct of terrestrial radio astronomy observations by avoiding interference and improving angular resolution. The paper also proposes a mechanism that enhances HPC utilisation. This paper makes the following contributions:

(1) It proposes an optimisation framework for terrestrial radio astronomy observations. The optimisation framework protects terrestrial radio astronomy observations from ISL interference and enhances the angular resolution of terrestrial radio astronomy organisation. The paper analyses additional data sets from the Karoo Array Telescope [19] to investigate the range of ISL transmit duration permissible without causing interference to GTs. The angular resolution is also enhanced by using CR enabled multimode GTs for terrestrial radio astronomy observations.

(2) It proposes an intelligent framework that uses similarities in astronomy source observation data for proactive interference avoidance between ISLs and GTs.

(3) The paper proposes the opportunistic computing scheme (OCS) that uses a duty cycle multiplex to enhance HPC utilisation. This paper investigates OCS's success probability and the terrestrial wireless network throughput as a function of the number of GTs.

The remainder of this paper is organised as follows. Section 2 discusses the related literature. Section 3 focuses on problem definition. Section 4 presents the proposed mechanisms. Section 5 discusses the simulation results. Section 6 concludes the paper.

\section{Related Work}

This section is divided into two parts. The first part addresses issues related to optimising terrestrial radio astronomy observations. It discusses literature focusing on the interference protection of GTs and improving angular resolution. The second part discusses the improvement of HPC utilisation.

\subsection{Optimising Terrestrial Radio Astronomy Observations.} Interference-free spectrum access and the improvement of angular resolution are important goals in the conduct of terrestrial radio astronomy observations. The goal of interference-free spectrum access can be achieved via spectrum reservation [20-24]. Spectrum reservation aims to ensure that new services do not encroach into bands dedicated for terrestrial radio astronomy observations. However, spectrum reservation faces interference challenges from new services which are ignorant of terrestrial radio astronomy observations. Interference mitigation measures such as restricting satellites from GT sky region are proposed in [24]. The solution in [24] does not consider the forwarding of data via ISLs through the satellite network. A restriction of satellites increases latency when the shortest path through the satellite network lies in the GT sky region. The proliferation of small satellite constellations [4-7] that use ISLs poses interference risks to GTs.

Another area of innovation in terrestrial radio astronomy observations is the conversion of unused earth stations to GTs. The increasing use of optical cables has been recognised to make some satellite earth stations redundant [9-14]. GTs realised from converted earth stations have been used in the UK [9, 10], Mozambique [11], Ghana [12, 13], and New Zealand [14]. The conversion of unused satellite earth stations enables the reuse of satellite installations and reduces astronomy infrastructure cost. However, the converted earth stations have been those without a tracking system.

It can be inferred from [9-14] that the satellite earth stations to be converted have been left unused for a long period 
of time. During the idle time of unused earth stations, the rollout of terrestrial wireless networks in concerned areas is not unlikely, thereby exposing GTs to terrestrial wireless network interference as inferred from [25-28]. Therefore, converted earth stations require interference protection mechanisms when they are in the vicinity of terrestrial wireless networks.

The use of additional earth stations alongside converted earth stations increases the number of GTs and the baseline. The increase in the number of GTs and baseline gives an opportunity to improve the angular resolution of terrestrial radio astronomy observation. In the absence of additional GTs, the terrestrial radio astronomy organisation has a fixed baseline. The angular resolution can be improved in a GT array with a dynamic baseline.

Furthermore, Woodburn et al. [14] recognise that the Goonhilly-3 GT can be used for satellite communications and terrestrial radio astronomy. Such a GT reduces costs due to dish and transponder reuse. A dual purpose GT can be opportunistically used to increase the baseline of terrestrial radio astronomy observations for a given period of time. However, the dual purpose GT intended for satellite communications and terrestrial radio astronomy observations requires mode switching mechanisms. The mode switching mechanism determines the epochs where the dual purpose GT can be used for receiving packets or radio astronomy data. The dual GT cannot be concurrently used for communications and astronomy observations. This is because the high transmit power in communication signals interferes with the astronomy source radio signal. The dual GT should also host mechanisms that protect it from terrestrial wireless network interference.

In addressing the challenges of interference mitigation and enhancing angular resolution, this paper considers the $\mathrm{CR}$ as suitable. CR spectrum sharing models are suitable for the design of interference protection schemes for terrestrial radio astronomy observations. In [29], the CR application considers the underlay spectrum sharing model and prevents interference by limiting terrestrial wireless network transmit power. A reduction of the terrestrial wireless network transmit power is proposed in [29] because astronomy source transmit power cannot be controlled. Investigations in [29] show that the desired coexistence between terrestrial wireless networks and GTs is infeasible. This is because of the large transmit power of terrestrial wireless networks compared to the very low received signal strength of astronomy source signals. However, the CR supports other spectrum sharing models such as the interweaving spectrum sharing model.

In addition, the CR benefits from the reconfigurable software defined radio. Being reconfigurable, the CR can be used to design a dual GT with a dynamic baseline. The inclusion of a dual GT in a manner that increases the baseline enhances observatory angular resolution. The inclusion of the CR in the dual GT enables the design of a GT that responds to the requirement of improving the angular resolution. Being suitable for designing solutions that enhance interference mitigation and angular resolution, the CR can be used to design technical solutions that enhance terrestrial radio astronomy observations [30]. However, the use of the CR in this aspect requires further consideration.
2.2. High Performance Computing Infrastructure Utilisation. The HPC is used to process the astronomy source radio waves that are received by GTs. It is connected to the GTs via optic fibre links. The terrestrial radio astronomy organisation should maximally utilise the HPC. Barbosa et al. [15] point out that the ALMA HPC has a 38\% utilisation as indicated in the ALMA Cycle 0 report. This results in low power efficiency, since the HPC is powered all the time [15].

The resulting HPC underutilisation can be addressed by using multiplexing techniques. Multiplexing techniques have been used in wireless communications for sharing bandwidth resources and suitable for enhancing HPC utilisation. The use of multiplexing proposed in [15] can also be extended to accommodate data processing from cognitive terrestrial wireless networks. Such an application can enhance the CR autonomous capability as seen in $[31,32]$. The CR in [31, 32] has a limited autonomous capacity because it does not autogenerate and train new learning mechanisms. Generative artificial intelligence [32] can enable the CR to autogenerate and train new intelligent mechanisms. The autogenerated learning mechanisms can be used to determine CR transmission parameters after training $[33,34]$. The discussion in $[31,32]$ has not considered using HPC's unused computational resources to train autogenerated learning mechanisms. Andreani [35] presents results that can be used to estimate the GT nonobservation time fraction in ALMA Cycle 3 spanning the period from October 2015 to August 2016. The discussion in [35] also identifies factors causing GT observation limitations such as opacity and phase stability. The occurrence of observation limitations affects GTs observation and is independent of interference from ISLs or terrestrial wireless networks. From the estimated observation fraction presented in [35], the ALMA HPC is left unutilised for $51.4 \%$ of the time that it is powered. Though, the ALMA underutilisation is noted to have reduced by $10.6 \%$, an underutilised capacity of $48.6 \%$ still exists.

\section{Problem Definition}

This section describes the challenges being addressed for a terrestrial radio astronomy organisation that uses GTs realised from converted unused earth stations. It is divided into two parts. The first part focuses on the optimisation goals. In the first part, the challenges discussed are those of interference avoidance and enhancing angular resolution. The second part describes the problem of enhancing HPC utilisation.

3.1. Optimisation: Defined Challenges for Terrestrial Radio Astronomy Observations. The considered scenario comprises low earth orbit satellites, GTs, cognitive base stations, and the HPC. The satellites are connected using ISLs. These entities have the following capabilities:

(1) Satellites: they are located in the low earth orbit and have a shortest path routing and station keeping algorithms

(2) High performance computing (HPC) infrastructure: the HPC is peta-scale and general purpose and is 
shielded from radio frequency interference. It allocates computational units to each GT and has access to high speed Internet links. The HPC can also determine when observation limitation conditions cause HPC underutilisation

(3) Ground telescopes (GTs): GTs are installed after launching the satellite constellation. They are connected to the HPC by optic fibre links. They present the observed astronomy source radio signals to the HPC. Each GT allocated HPC computational units. The allocated HPC computational units are used to process signals received from the GT

(4) Cognitive base station (CBS): the CBS is a massive multiantenna system and is the central entity in terrestrial wireless network. It incorporates generative artificial intelligence, autogenerates learning mechanisms, and is connected to the HPC via high speed Internet links. The CBS uses the orthogonal frequency division multiplex-space division multiple access technology and receives signal streams from multiple terrestrial wireless network subscribers. Individual subscriber signals are extracted from the multiplexed signal by an artificial neural network multiuser detector. The multiuser detection aims to reduce user bit error rate. The CBS receives subscriber bit error rate via the control channel. It compares subscriber bit error rate with a predefined bit error rate threshold. The CBS autogenerates new artificial neural network multiuser detectors when user bit error rate exceeds the bit error rate threshold. It keeps existing artificial neural network multiuser detectors and examines their suitability in different future contexts

Let $\alpha, G, \phi$, and $\theta$ denote the set of satellites, GTs, HPC computational units allocated to GTs, and the utilisation of HPC computational units allocated to GTs, respectively.

$$
\begin{aligned}
& \alpha=\left\{\alpha_{1}, \ldots, \alpha_{i}\right\}, \\
& G=\left\{G_{1}, \ldots, G_{m}\right\}, \\
& \phi=\left\{\phi_{1}, \ldots, \phi_{m}\right\}, \\
& \theta=\left\{\theta_{1}, \ldots, \theta_{m}\right\},
\end{aligned}
$$

where $i$ and $m$ are the maximum numbers of ISLs and GTs, respectively.

In addition, let $\eta, v, f_{\eta}$, and $f_{G}$ be the sets of satellite sky region, GT sky region, ISL frequency, and GT frequency, respectively.

$$
\begin{gathered}
\eta=\left(\eta_{1}, \ldots, \eta_{i}\right), \\
v=\left(v_{1}, \ldots, v_{m}\right), \\
f_{\eta}=\left(f_{\eta_{1}}, \ldots, f_{\eta_{i}}\right), \\
f_{G}=\left(f_{G_{1}}, \ldots, f_{G_{m}}\right) .
\end{gathered}
$$

Satellites that are interconnected via ISLs and in the sky region of GTs cause intermodulation interference to

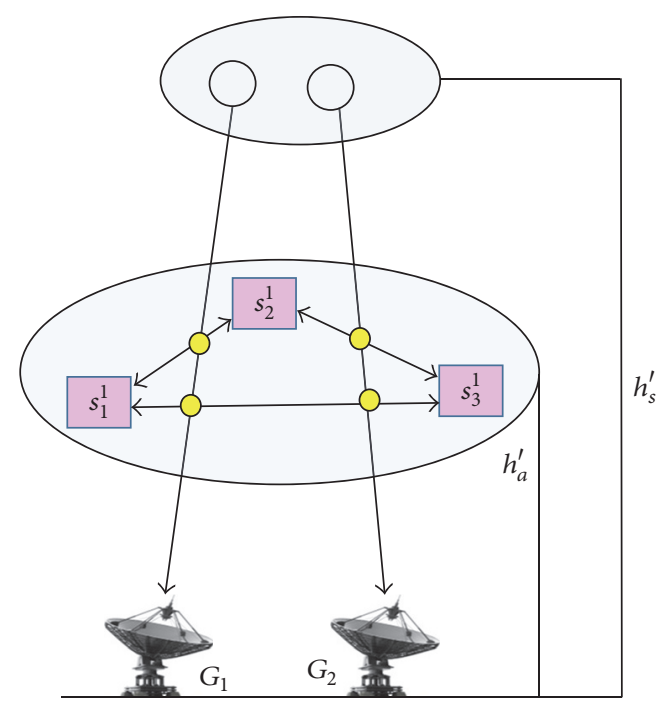

FIGURE 1: Interference between ISLs and GBTs.

terrestrial radio astronomy observations. The spectral main and side band signals are transmitted in the ISL antenna main lobe and side lobes, respectively. The presence of multiple satellites in the low earth orbit leads to the existence of multiple side lobes alongside each ISL main lobe due to nonideal satellite side lobe suppression. The ISL signal interferes with the astronomy source radio signal because the ISL's main and side lobe signals have a higher power than the astronomy source radio signal power.

Interference arises between ISLs and GTs when $v_{c}=\eta_{d}$, $f_{G_{c}}=f_{\eta_{d}}, c \in\{1, \ldots, m\}$, and $d \in\{1, \ldots, i\}$. A scenario showing the occurrence of an interference point can be seen in Figure 1. In Figure 1, astronomy sources and satellites have altitudes, $h_{a}^{\prime}$ and $h_{s}^{\prime}$, respectively, where $h_{a}^{\prime}>h_{s}^{\prime}$. The operating frequencies of GTs, $G_{1}, G_{2}$, are $f_{G_{1}}$ and $f_{G_{2}}$, respectively. The satellite sky region has three satellites, $s_{1}^{1}, s_{2}^{1}$, and $s_{3}^{1}$, which are connected with ISLs. ISLs $s_{1}^{1}-s_{2}^{1}, s_{2}^{1}-s_{3}^{1}$, and $s_{1}^{1}-s_{3}^{1}$ transmit on $f_{\eta_{1}}, f_{\eta_{2}}$, and $f_{\eta_{3}}$, respectively. The interference point in Figure 1 arises via either additive or multiplicative or other combinations of radio astronomy and the ISL signals.

The interference arises because of the nonlinear combination of ISL and astronomy source signals. ISL signals have intermodulation products that are radiated through the satellite antenna's main and side lobes. The radiated signals comprise intermodulation products and have a higher power than the astronomy source signals. The stronger ISL radiated signals cause interference with the weaker astronomy source signal. In the problem formulation here, the paper aims to demonstrate that ISL signals cause interference to astronomy source signals. The ISL signal is recognised to comprise multiple intermodulation products; the aim here is not to list these products but to demonstrate that their presence interferes with terrestrial radio astronomy organisations.

Let $\psi_{\alpha_{a}, G_{c}}^{\eta}$ denote the ISL signal from satellite $\alpha_{a}, a \in$ $\{1, \ldots, i\}$, traversing $\eta$ over $G_{c}$. In addition, let $\gamma_{p}^{v}$ denote the astronomy source radio signal $p$ received by $G_{c}$ in $v$, 
respectively. In the absence of interference protection, the GT receives the signal, $s_{p, v_{c}}^{\alpha_{a}, G_{c} \eta_{d}}$, given as

$$
\begin{aligned}
s_{p, v_{c}}^{\alpha_{a}, G_{c}, \eta_{d}}= & A+B, \\
A= & \sum_{a=1}^{i} \sum_{c=1}^{m} \psi_{\alpha_{a}, G_{c}}^{\eta_{a}}+\sum_{p=1}^{P} \sum_{c=1}^{m} \gamma_{p}^{v_{c}}+\prod_{a=1}^{i} \prod_{c=1}^{m} \psi_{\alpha_{a}, G_{c}}^{\eta_{a}} \\
& \times \prod_{p=1}^{P} \prod_{c=1}^{m} \gamma_{p}^{v_{c}}, \\
B= & \sum_{a=1}^{i} \sum_{c=1}^{m} \psi_{\alpha_{a}, G_{c}}^{v_{c}}+\prod_{p=1}^{P} \prod_{c=1}^{m} \gamma_{p}^{v_{c}}+\prod_{a=1}^{i} \prod_{c=1}^{m} \psi_{\alpha_{a}, G_{c}}^{\eta_{a}} .
\end{aligned}
$$

$A$ and $B$ are intermodulation products arising from the additive and multiplicative components of $\psi_{\alpha_{a}, G_{c}}^{\eta}$ and $\gamma_{p}^{v}$. The additive and multiplicative components arise due to the combinations of different ISL signal components alongside components of the astronomy source radio signal.

Furthermore, let $N_{G}(t), D(G, t)$, and $\lambda_{G}(t)$ denote the sets of (1) terrestrial wireless networks in GT vicinity at time $t$, (2) GTs baseline at time $t$, and (3) GT observation wavelength at time $t$, respectively.

$$
\begin{aligned}
t & =\left\{t_{1}, \ldots, t_{r}\right\}, \\
N_{G}(t) & =\left\{N_{G_{1}}(t), \ldots, N_{G_{j}}(t)\right\}, \\
D(G, t) & =\left\{\left(D\left(G_{1}, t\right)\right), \ldots,\left(D\left(G_{m}, t\right)\right)\right\}, \\
\lambda_{G}(t) & =\left\{\lambda_{G_{1}}(t), \ldots, \lambda_{G_{m}}(t)\right\} .
\end{aligned}
$$
GTs is

The angular resolution, $R\left(m-4, t_{1}\right)$, at time $t_{1}$ given $(m-4)$

$$
R\left(m-4, t_{1}\right)=\sum_{c=1}^{m-4} \frac{\left(\lambda_{G_{c}}, t_{1}\right)}{\max \left(D\left(G_{c}, t_{1}\right)\right)} .
$$

Given that the baseline can be increased by using an additional GT such that $\max \left(D\left(G_{m-3}, t_{1}\right)\right)>\max \left(D\left(G_{m-4}, t_{1}\right)\right)$, the angular resolution, $R\left(m-3, t_{1}\right)$, is

$$
R\left(m-3, t_{1}\right)=\sum_{c=1}^{m-3} \frac{\left(\lambda_{G_{c}}, t_{1}\right)}{\max \left(D\left(G_{c}, t_{1}\right)\right)} .
$$

However, the terrestrial astronomy organisation requires an algorithm that enables it to increase its baseline by using an additional GT. The access to the additional GT ensures that $\max \left(D\left(G_{m-3}, t_{1}\right)\right)>\max \left(D\left(G_{m-4}, t_{1}\right)\right)$ without constructing a new GT. In addition, let $f_{G}(t)$ be the set of terrestrial wireless network frequencies in GT vicinity:

$$
f_{G}(t)=\left\{f_{G_{1}}(t), \ldots, f_{G_{j}}(t)\right\},
$$

where $j$ is the maximum number of terrestrial wireless network channels. Interference arises between the terrestrial wireless network and GTs when $f_{G_{j^{\prime}}}(t)=1 / \lambda_{G_{c}}(t)$,

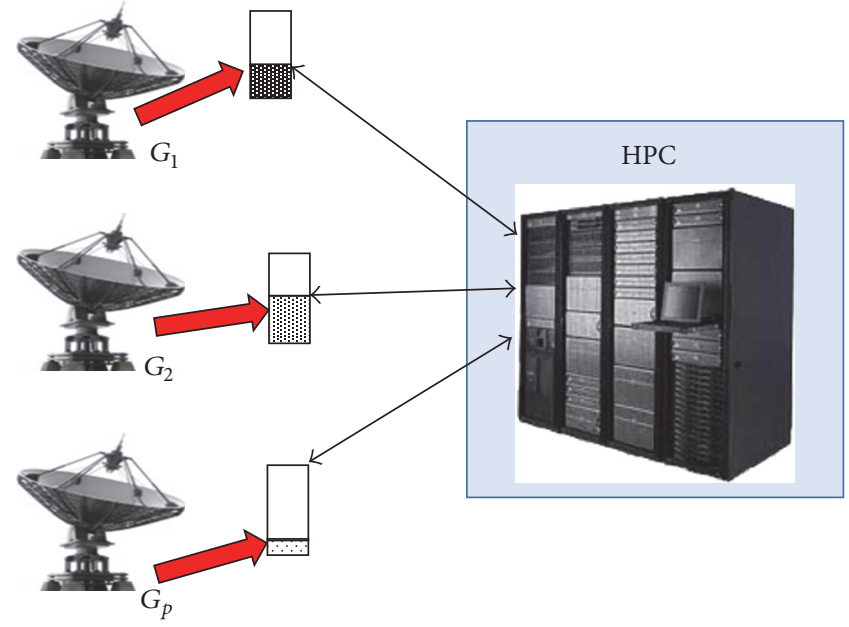

17.7.7. $\mathrm{HPC}$ utilisation between $12 \%$ and $50 \%$

$\square$ HPC utilisation between $50 \%$ and $80 \%$

HPC utilisation less than $12 \%$

$\Longrightarrow$ Optic fibre link (OFL)

$\longleftrightarrow$ Allocated computational units on HPC

FIGURE 2: HPC underutilisation.

$j^{\prime} \in\{1, \ldots, j\}$. Hence, GTs require an interference protection mechanism.

The discussion above has not considered the processing of the signals received by each GT. Each GT allocated $\phi_{c}$ HPC computational units, each having utilisation $\theta_{c}$. The relations between GTs and the HPC for a case of HPC underutilisation and near optimal utilisation are shown in Figures 2 and 3, respectively.

Figure 2 shows the case where $p, p \in\{1, \ldots, m\}$, GTs utilise the HPC. The first, second, and pth GTs have utilisation lying within $12 \%$ to $50 \%$ to $80 \%$ and less than $12 \%$, respectively. In this case, $\phi_{1 \text {,res }}$ HPC computational units are unutilised.

Figure 3 shows the case where $p$ GTs have near optimal HPC utilisation because all GTs utilise their computational units by up to $80 \%$. In this case, HPC's underutilised computational unit is $\phi_{2 \text {,res }}$ and $\phi_{2 \text {,res }}<\phi_{1 \text {,res }}$.

The scenario given in Figure 2 can be described as

$$
\begin{aligned}
\theta_{2} & >\theta_{1}>\theta_{p}, \\
\phi_{1} & =\phi_{2}=\phi_{p}, \\
\sum_{p=1}^{p} \phi_{p}\left(1-\theta_{p}\right) & =\phi_{1, \mathrm{res}} .
\end{aligned}
$$

The scenario given in Figure 3 can be described as

$$
\begin{aligned}
\theta_{2} & =\theta_{1}=\theta_{p}, \\
\phi_{1} & =\phi_{2}=\phi_{p}, \\
\sum_{p=1}^{p} \phi_{p}\left(1-\theta_{p}\right) & =\phi_{2, \text { res }} .
\end{aligned}
$$




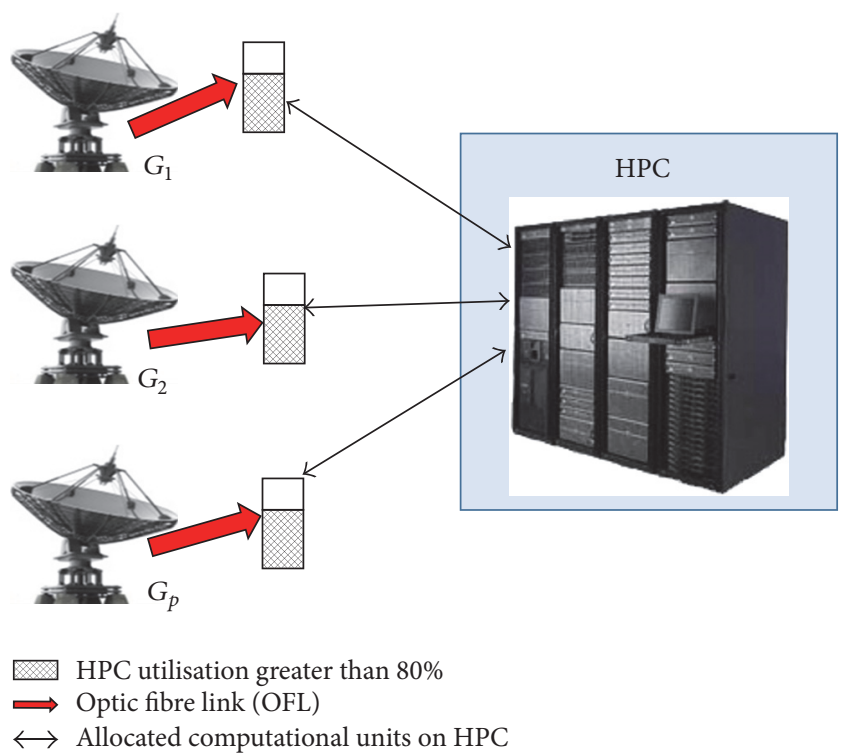

Figure 3: Near optimal HPC utilisation.

Given that $\phi_{\text {th }}$ is the threshold computational unit required for HPC sharing, the underutilised HPC can be shared with external applications if $\phi_{1 \text {,res }}<\phi_{\text {th }}$ or $\phi_{2 \text {,res }}<$ $\phi_{\text {th }}$. However, the HPC requires a mechanism to verify when $\phi_{1, \text { res }}<\phi_{\text {th }}$ or $\phi_{2, \text { res }}<\phi_{\text {th }}$.

\section{Proposed Schemes for Enhancing Terrestrial Radio Astronomy Observation}

This section presents the proposed schemes and consists of two parts. The first part discusses the optimisation schemes. The optimisation scheme protects terrestrial radio astronomy observations from ISL interference and enhances angular resolution. The second presents the opportunistic computing scheme (OCS) proposed to enhance HPC utilisation.

The proposed schemes incorporate the CR that is reconfigurable and can make decisions for different contexts of a given application. Though most CR applications focus on terrestrial wireless networks, CR capabilities can enhance terrestrial radio astronomy observations goals. The CR acquires environmental awareness via sensing, makes inferences using sensed results, determines reconfiguration options, and executes the reconfiguration decisions.

4.1. Proposed Optimisation Mechanisms. The optimisation of terrestrial radio astronomy observations aims to achieve interference mitigation and enhance angular resolution. In this paper, we propose a CR interference mitigation framework that extends [8] by considering the similarities in the observation order of astronomy sources.

The interference mitigation framework is located on the satellite, assumes that the astronomy organisation has a database of the epochs of previously observed astronomy sources, and comprises three entities. The entities are as follows:
(1) Cognitive reasoner (CRE): the cognitive reasoner receives two sets of information from the terrestrial radio astronomy organisation. The first set comprises the right ascension RA, declination $D$, observation frequency $\mathrm{OF}$, duration $\mathrm{ODu}$, and dates ODt. These are held in the tuple (RA, D, OF, ODu, ODt). The second set comprises similarly observed sources, $G$ with right ascension RA, and total observation duration $D$. The information on RA and $D$ is held in $S$. The information on $G, S$, and $I$ is held in the tuple $(G, S, I)$. The CRE uses the information in (RA, $D, \mathrm{OF}, \mathrm{ODu}, \mathrm{ODt})$ and $(G, S, I)$ to determine the ISL activation epochs and duration. The tuples (RA, D, OF, ODu, ODt) and $(G, S, I)$ are the first and second tuples, respectively

(2) Cognitive ISL deactivator (CSLA): the CSLA receives CRE outputs and uses these to determine the ISL transmission status and duration

(3) Plan acquisition channel (PAC): the PAC is a control channel that enables communications between the satellite's CRE and the terrestrial radio astronomy organisation. The first and second tuples are transmitted to the CRE from the terrestrial radio astronomy organisation via the PAC

The consideration of similarities implies that satellite does not have to analyse similar patterns all the time, thereby increasing ISL duration without interfering with GTs. The satellite does not have to analyse similar patterns all the time. Therefore, using similarity information can prevent interference to ongoing terrestrial radio astronomy observations while increasing ISL transmit duration.

The framework's flowchart is shown in Figure 4. As shown in Figure 4, the astronomy organisation transmits the first and second tuples via the PAC to the satellite via the PAC prior to commencing an observation. The CRE receives the first and second tuples and analyses them to determine the interference free ISL transmit epochs and duration. The CRE also determines whether a new similar pattern of observed sources is in the data received via the PAC. The new similar pattern is then used to update the second tuple on the satellite. The CSLA receives the CRE outputs and uses them to configure the ISL transmission status and duration.

Besides interference protection, optimising terrestrial radio astronomy observations also requires enhancing angular resolution. CR's reconfigurability is a useful feature in this regard. This paper applies a CR user classification to terrestrial radio astronomy observations. Terrestrial radio astronomy observations can be conducted using either primary GTs or secondary GTs. A primary GT is a GT that is designed for terrestrial radio astronomy observations only. The secondary GT is a GT that is capable of multiple applications. It can be used for other applications besides terrestrial radio astronomy observations. The Goonhilly-3 GT [14] intended for satellite communications and radio astronomy observations is an example of a secondary GT. This paper extends [14] by considering the CR as being suitable for designing a secondary GT. 


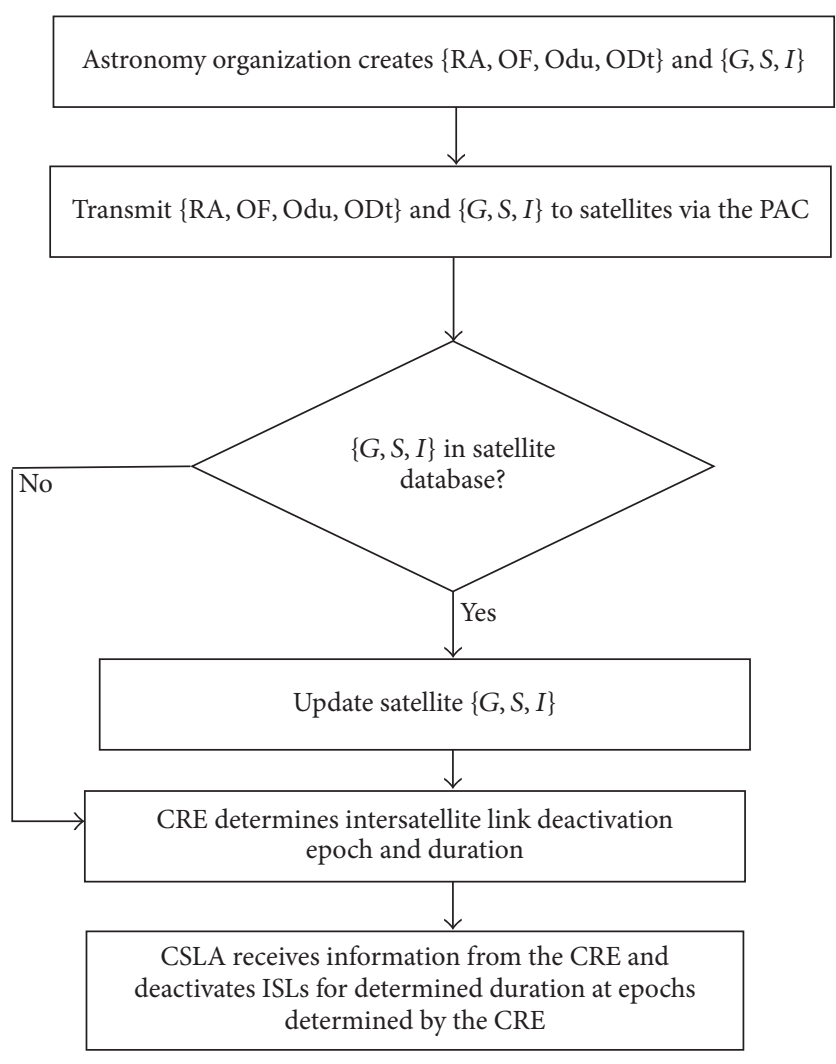

FIgURE 4: Flowchart of the learning framework.

The secondary GT incorporates a CR with mode switching and spectrum sensing mechanisms. The mode switching mechanisms enable the secondary GT to process communication packets and radio astronomy signals. This paper proposes a framework that enables interactions between terrestrial radio astronomy organisations (with primary GTs) and secondary GTs. Primary and secondary GTs interact via the Internet. Secondary GTs belong to other organisations. Radio astronomy data observed by the secondary GT are transmitted to the terrestrial radio astronomy organisation via the Internet.

The framework proposed to enhance the angular resolution has two ends belonging to the terrestrial astronomy organisation and the organisation that owns the secondary GTs. Each end has two entities. The entities at the terrestrial radio astronomy organisations are as follows:

(1) Astronomy data processor (ADP): the ADP processes radio astronomy data from primary and secondary GTs

(2) Satellite astronomy interface (SAI): the SAI holds information on the terrestrial radio astronomy organisation's observation objectives. It accesses accessible secondary GTs information capability via the Internet

The entities at the organisation that owns the secondary GTs are as follows:

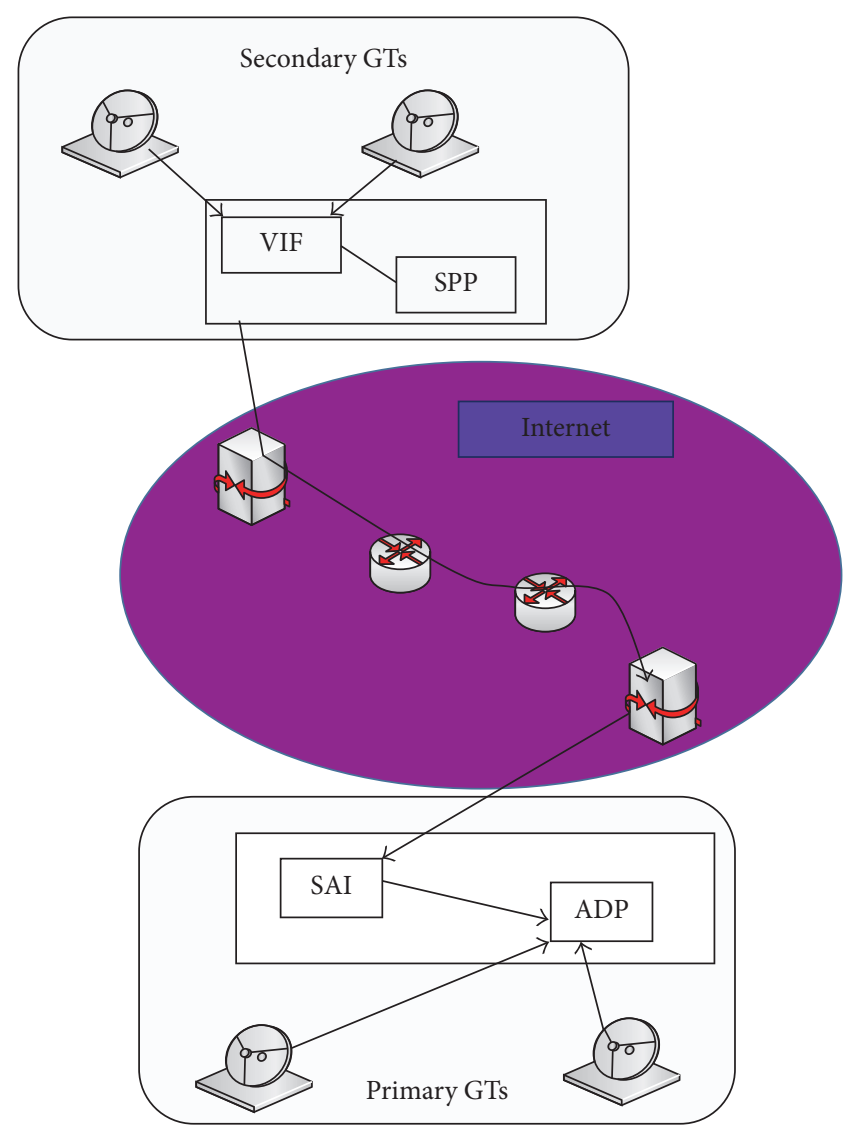

FIGURE 5: Interaction between primary and secondary GT.

(1) Visibility interface (VIF): the VIF collates information on the satellite visibility epoch and duration. The VIF accesses radio astronomy observation requirements from the SAI and determines suitable secondary GTs. In addition, the VIF obtains radio astronomy data from secondary GTs that are used for terrestrial radio astronomy observations. The obtained data is sent to the SAI via the Internet. The SAI sends radio astronomy data received from the VIF to the ADP

(2) Satellite packet processor (SPP): the SPP processes satellite communication packets when secondary GTs are used for satellite communications.

In this paper, the secondary GTs are owned by satellite communication network operators.

Relations between the ADP, SAI, VIF, and SPP are shown in Figure 5. In Figure 5, the secondary GT's packets and satellite visibility epochs are transmitted to the VIF. The VIF forwards packets to the SPP. It obtains and transmits satellite visibility epochs to the SAI via the Internet. CES data are processed at the ADP. Secondary GT's astronomy data are also sent to the ADP via the Internet. The SAI is aware of terrestrial radio astronomy observation goals and checks the SVB for available secondary GTs using SAI information. A bidirectional link exists between the SAI and the VIF. 
Data obtained from the SAI are used by the VIF to select secondary GTs that satisfy the terrestrial radio astronomy organisation's observation objectives. The selection is done at the VIF. The selection algorithm considers the desired observation longitude, $L_{s}^{v}$, latitude, $l_{s}^{v}$, and frequency, $f_{v}$, of $v$. The information on $L_{s}^{v}, l_{s}^{v}$, and $f_{v}$ is held in $T_{1}^{v}$. The selection procedure considers the $z$ th secondary GT's longitude, $L_{e}^{z}$, latitude, $l_{e}^{z}$, and CR frequencies, $f_{e}^{z}$. Information on $L_{e}^{z}, l_{e}^{z}$, and $f_{e}^{z}$ is held in $T_{2}^{z}$. The SAI selects the secondary GTs for which the Euclidean distance $d\left(T_{1}^{v}, T_{2}^{z}\right)$ is minimum.

$$
\begin{aligned}
& d\left(T_{1}^{v}, T_{2}^{z}\right) \\
& =\min \sum_{c=1}^{m} \sum_{z=1}^{V} \sqrt{\left(L_{s}^{v_{c}}-L_{e}^{z}\right)^{2}+\left(l_{s}^{v_{c}}-l_{e}^{z}\right)^{2}+\left(f_{v_{c}}-f_{e}^{z}\right)^{2}} .
\end{aligned}
$$

The secondary GT hosts a cyclostationary detector that can perfectly differentiate between radio astronomy and terrestrial wireless network signals. The perfect differentiation is achieved because terrestrial wireless networks use modulation schemes with known cyclostationary signatures. The secondary GT stops radio astronomy observations when terrestrial wireless signals are detected. The probability $P_{c}\left(v, z, d\left(T_{1}^{v}, T_{2}^{z}\right)\right)$ that using $z$ secondary GTs helps the terrestrial radio astronomy organisation to realise its observation objectives is

$$
P_{c}\left(v, z, d\left(T_{1}^{v}, T_{2}^{z}\right)\right)=1-e^{-(v+z) d\left(T_{1}^{v}, T_{2}^{z}\right)} .
$$

The use of secondary GTs also enhances the angular resolution. A smaller angular resolution is more beneficial. The angular resolution, $\theta_{1}$, when the terrestrial radio astronomy organisation does not use secondary GTs is

$$
\theta_{1}=\sum_{c=1}^{m} \frac{\lambda_{c}}{b_{\max }}
$$

where $\lambda_{c}$ is the observation wavelength of the $c$ th GT and $b_{\max }$ is the observatory baseline.

In the case where there are terrestrial wireless networks in the vicinity of primary and secondary GTs, primary and secondary GTs experience interference. The resulting interference reduces the baseline's contribution in enhancing the angular resolution. The angular resolution is degraded because the electromagnetic radiation pattern of the terrestrial wireless network infiltrates that of the astronomical source being received by GTs. Given that the baseline is reduced by $\omega$, the angular resolution $\theta_{2}$ when secondary GTs are not incorporated and primary GTs experience terrestrial wireless network interference is

$$
\theta_{2}=\sum_{c=1}^{m} \frac{\lambda_{c}}{b_{\max }(1-\omega)}
$$

The use of secondary GTs alongside primary GTs improves the angular resolution, while using additional secondary GTs increases the baseline. In this case, the baseline is increased by $\alpha^{\prime}$. Assuming that both primary and secondary GTs are unaffected by terrestrial wireless network interference, the angular resolution $\theta_{3}$ is

$$
\theta_{3}=\sum_{c=1}^{m} \frac{\lambda_{c}}{b_{\max }\left(1+\alpha^{\prime}\right)}+\sum_{z=1}^{B^{\prime}} \frac{\lambda_{z}}{b_{\max }\left(1+\alpha^{\prime}\right)}
$$

where $\lambda_{z}$ is the $z_{\text {th }}$ secondary GT's observation wavelength and $B^{\prime}$ is the maximum number of secondary GTs.

In the event that primary and secondary GTs have terrestrial wireless networks in their vicinity. it is considered that primary and secondary GTs do not incorporate and incorporate an ideal cyclostationarity detector, respectively. The angular resolution, $\theta_{4}$, is

$$
\theta_{4}=\sum_{c=1}^{m} \frac{\lambda_{c}}{b_{\max }\left(1+\alpha^{\prime}\right)(1-\omega)}+\sum_{z=1}^{B^{\prime}} \frac{\lambda_{z}}{b_{\max }\left(1+\alpha^{\prime}\right)} .
$$

4.2. Opportunistic Computing Scheme (OCS). The proposed OCS is a synergy between the CBS and HPC. The CBS uses artificial neural network multiuser detectors to ensure low bit error rate signal reception. The multiuser detectors are developed by training the neural networks with different bits of known modulated signals and user bit patterns for different channel states and multiantenna configuration. The cyberphysical system has two Internet entities that interact with the CBS and the HPC. These entities are as follows:

(1) Neural resource monitor (NRM): the NRM monitors CBS's usage of computational resources. It determines when the CBS resources are insufficient for developing newly autogenerated artificial neural network multiuser detectors. The NRM receives training data and instructions from the CBS and sends them to the TRM

(2) Training resource monitor (TRM): the TRM receives CBS training data and instructions from the NRM. It sends CBS training data and instructions of artificial neural network multiuser to underutilised HPC. The HPC executes CBS training instructions when observation limitation conditions results in HPC underutilisation by the GTs.

The OCS system showing relations between the CBS and the HPC at epochs $t_{1}$ and $t_{2}$ is shown in Figure 6. The CBS autogenerates new artificial neural network multiuser detectors when the obtained bit error rate exceeds the predefined threshold.

As shown in Figure 6, the CBS initially has four artificial neural network multiuser detectors, that is, brain like learning mechanisms at epoch $t_{1}$. The achieved bit error rate exceeds the predefined threshold bit error rate at epoch $t_{1}$. Hence, the development of new artificial neural network multiuser detectors is required. The CBS autogenerates new artificial neural network multiuser detectors at $t_{2}$. However, at $t_{2}$, the CBS does not have sufficient computational units to train the two autogenerated artificial neural network multiuser detectors. The OCS success probability is formulated using the probability that there are sufficient HPC computational 


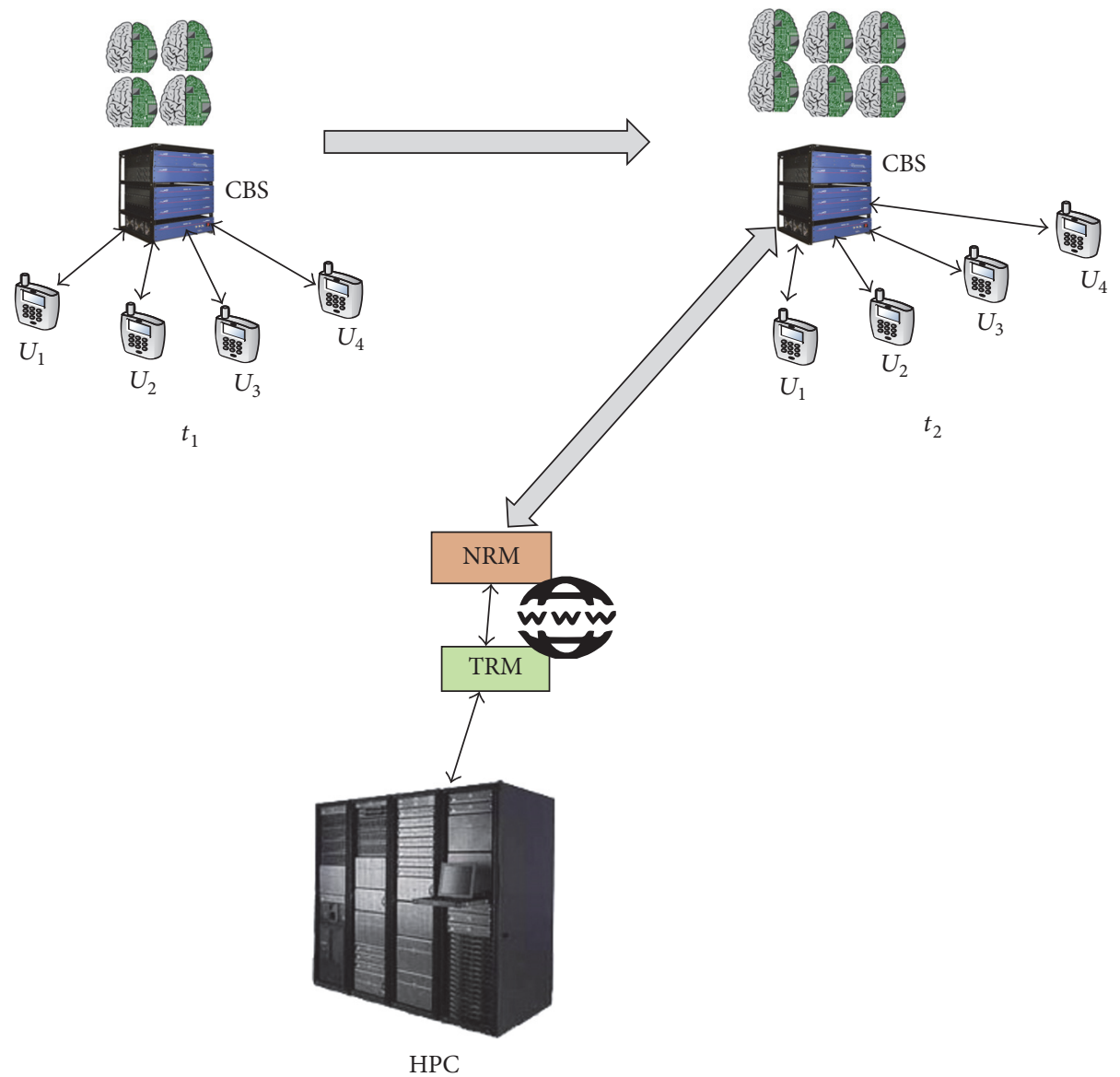

FIGURE 6: Evolution of states of artificial neural network multiuser detectors at epochs $t_{1}$ and $t_{2}$.

units for $c$ GTs at time $t, P_{c}(c, t)$, and the probability of CCM failure for $c$ telescopes at time $t, P_{a}(c, t)$. OCS fails in the following cases:

(1) There is TRM failure incident, though there are sufficient HPC computational resources.

(2) The TRM does not fail but there are insufficient HPC computational resources.

The OCS success probability, $P_{\mathrm{OCS}}(c, t)$, can be obtained as follows:

$$
\begin{aligned}
& P_{\mathrm{OCS}}(c, t)=1-\left(P_{a}(c, t) \times P_{c}(c, t)+\left(1-P_{a}(c, t)\right)\right. \\
& \left.\quad \times\left(1-P_{c}(c, t)\right)\right) .
\end{aligned}
$$

$P_{\mathrm{OCS}}(c, t)$ is evaluated using the newly modified Weibull function [36] to model $P_{c}(c, t)$ and $P_{a}(c, t)$. OCS influences terrestrial wireless network throughput. The throughput is formulated to investigate OCS's ability to develop an artificial neural network multiuser detector that enhances signal reception by reducing the bit error rate when executing multiuser detection. A high OCS success execution probability results in a multiuser detector that reduces the number of corrupted bits received per second by the terrestrial wireless network subscriber. The reduction in the number of received corrupted bits increases the number of noncorrupted bits received per second by each subscriber, thereby enhancing terrestrial wireless network throughput.

In formulating the terrestrial wireless network throughput when OCS is used, we consider a scenario where CRs transmit to the CBS with transmit power, $p_{11}^{\mathrm{tr}}$, over a channel with gain, $h_{11}^{\text {tr }}$. The transmitting CR experiences interference from neighbouring users. The interfering channel's gains and powers are given as $\left(h_{12}^{\text {int }}, h_{13}^{\text {int }}, \ldots, h_{1 n}^{\text {int }}\right)$ and $\left(p_{12}^{\text {int }}, p_{13}^{\text {int }}, \ldots, p_{1 n}^{\text {int }}\right)$, respectively. The development of an ideal artificial neural network multiuser detector occurs when $P_{\mathrm{OCS}}=1$; when $P_{\mathrm{OCS}}<1$, the artificial neural network multiuser detector is nonideal due to interference effects. Shannon throughput $C$ when the terrestrial wireless network subscriber transmits on one channel is

$$
C=\log _{2}\left(1+\frac{\left(h_{11}^{\mathrm{tr}}\right)^{2} \times p_{11} \times P_{\mathrm{OCS}}}{\sum_{n^{\prime}=1}^{n}\left(h_{1 n^{\prime}}^{\mathrm{int}}\right)^{2} \times p_{1 n^{\prime}}^{\mathrm{int}}}\right) .
$$

The subscriber can also use multiple channels with each channel having own interfering subscribers. The use of artificial neural network multiuser detectors developed via OCS reduces the number of received corrupted bits and enhances the signal to interference ratio, thereby improving throughput. Let $P_{\mathrm{OCS}}^{n^{\prime}}$ be the probability of successfully executing OCS for channel $n^{\prime}$. In addition, let $h_{11}^{n^{\prime}}$ and $p_{11}^{n^{\prime}}$ be 
TABLE 1: Analysis results of the spectrum utilisation and transmit opportunity.

\begin{tabular}{lcccc}
\hline S/N & Observation day & Observation duration & Spectrum utilisation (\%) & Transmit opportunity (\%) \\
\hline 1 (considered in [8]) & $16 / 02 / 13$ & 28740 & 33.3 & 66.7 \\
2 (considered in [8]) & $21 / 01 / 13$ & 14700 & 17 & 83 \\
3 & $14 / 10 / 12-15 / 10 / 12$ & 31080 & 36 & 64 \\
4 & $16 / 10 / 12$ & 15180 & 17.57 & 82.43 \\
5 & $28 / 10 / 12$ & 36931 & 42.74 & 52.76 \\
6 & $06 / 11 / 12-07 / 11 / 12$ & 40800 & 57.22 & 42.78 \\
7 & $14 / 11 / 12-15 / 11 / 12$ & 36119 & 41.80 & 58.20 \\
8 & $05 / 02 / 13$ & 9846 & 11.36 & 88.64 \\
9 & $11 / 02 / 13$ & 18740 & 21.74 & 78.26 \\
10 & $23 / 02 / 13$ & 21730 & 25.15 & 74.85 \\
\hline
\end{tabular}

the CR transmit channel gain and power over $n^{\prime}$, respectively. Similarly, let $h_{1 i^{\prime}}^{n^{\prime}}$ and $p_{1 i^{\prime}}^{n^{\prime}}$ be the interfering channel gain and power of user $i^{\prime}$ over $n^{\prime}$. The throughput, $C_{1}$, when the CR transmits over $N^{\prime}$ channels, each of capacity $B \mathrm{~Hz}$, is

$$
C=N^{\prime} B \log _{2}\left(1+\frac{\sum_{n^{\prime}=1}^{N^{\prime}}\left(h_{11}^{n^{\prime}}\right)^{2} \times p_{11}^{n^{\prime}} \times P_{\mathrm{OCS}}^{n^{\prime}}}{\sum_{n^{\prime}=1}^{N^{\prime}} \sum_{i^{\prime}=1}^{m^{\prime}}\left(h_{1 i^{\prime}}^{n^{\prime}}\right)^{2} \times p_{1 i^{\prime}}^{n^{\prime}}}\right) .
$$

\section{Performance Investigation}

This section discusses the simulation results for the proposed mechanisms. It is divided into two parts. The first part presents results of terrestrial radio astronomy observation optimisation. It presents results on the spectrum usage analysis, similar observation strings of astronomy sources, and angular resolution. The second part investigates OCS's success execution probability and how OCS enhances terrestrial wireless network throughput.

5.1. Spectrum Usage Analysis. This section presents results on the spectrum usage of radio astronomy observations and examines ISL back-to-back duration and similar astronomy source observation strings. The spectrum utilisation and transmit opportunities are computed for the following days: $21 / 01 / 13,16 / 02 / 13,14 / 10 / 12,15 / 10 / 12,16 / 10 / 12,28 / 10 / 12$, $07 / 11 / 12,14 / 11 / 12,15 / 11 / 12,05 / 02 / 13,11 / 02 / 13,23 / 02 / 12$, and $06 / 11 / 12$. The data used for $06 / 11 / 12$ in [8] describes observations conducted between 17:15:54.8 and 23:37:42.6, while here it concerns observation made between 16:12:24.8 and 23:59:45.2. Data analysis results are shown in Table 1.

The average spectrum utilisation is computed using data in [8] and is also recalculated using new data. It is estimated to be $25.6 \%$ for data solely used in [8] and $29.1 \%$ when new data is incorporated, respectively. The average spectrum utilisation of radio astronomy observation increases by $3.5 \%$ when additional data is incorporated. The transmit opportunity $Y$ decreases by $3.5 \%$. The observation day, observation duration, spectrum utilisation, and transmit opportunity for previously considered data sets and the additional eight samples are presented in Table 1.
The standard deviation before and after the inclusion of more data for generalisation is evaluated to be $8.2 \%$ and $11.9 \%$, respectively. Hence, the daily transmit duration is approximately one-ninth of the maximum obtained transmit opportunities. These transmit opportunities are exclusive of opportunities existing during the conduct of radio astronomy observations.

We also analyse the back-to-back duration $D$ to determine the ISL transmit duration, while terrestrial radio astronomy observations are ongoing. The ISL transmit opportunities arise due to observation switching events. Switching occurs when the astronomy organisation has just finished observing an astronomy source and is about to commence the observation of another source. The switching results in a period during which terrestrial radio astronomy observations are not conducted. These periods are potential interferencefree ISL transmit opportunities and are repeated for astronomy source observation patterns. The intelligent framework is used to determine the transmit duration.

In analysing $D$, we use extra data for observations conducted on 06/11/12 (period 1) and 07/11/12 (period 2) in addition to that of 16/02/13 (period 3) used in [8]. The observation in periods 1, 2, and 3 has data for 30,85 , and 95 observation epochs, respectively. In presenting data analysis results, the periods are classified as (1) early morning, 00:00:00.006:00:00.0, (2) morning, 08:42:05.3-12:02:55.5, (3) mid afternoon, 12:02:55.6-15:07:5.7, and (4) late afternoon, 15:07:15.818:34:55.7. The observation durations in these epochs are shown in Table 2.

The plots for the back-to-back connection for observations conducted in periods 1, 2, and 3 are shown in Figures 7, 8, and 9, respectively. From the results in Figures 7, 8, and 9, it can be observed that the analysis of additional data shows that ISL transmission can benefit from transmit opportunities due to the back-to-back connection duration. Further analysis shows that the average ISL back-to-back connection duration for periods 1, 2, and 3 is 49.5 seconds, 58.8 seconds, and 43.7 seconds, respectively.

The similar astronomy source observations strings for different observation dates are as follows:

(1) Source string 1: $a_{1}=$ PKS 1934-638, $b_{1}=$ PKS J00104153, $c_{1}=$ PKS J0022 $+0014, d_{1}=$ PKS J0024-4202, 
TABLE 2: Observation epochs used to investigate the back-to-back connection duration.

\begin{tabular}{|c|c|c|c|c|}
\hline $\begin{array}{l}\text { Observation } \\
\text { epochs/periods }\end{array}$ & Early morning & Morning & Mid afternoon & Late afternoon \\
\hline Period 1,85 epochs & & & & $00: 01: 20.2-02: 52: 35.3$ \\
\hline \multicolumn{5}{|l|}{$\begin{array}{l}\text { Period 2, } 95 \text { epochs, } \\
\text { 00:01:20.2-02:52:35.3 }\end{array}$} \\
\hline Period 3, 95 epochs & $08: 42: 05.3-12: 02: 55.5$ & $12: 02: 55.6-15: 07: 15.7$ & & \\
\hline
\end{tabular}

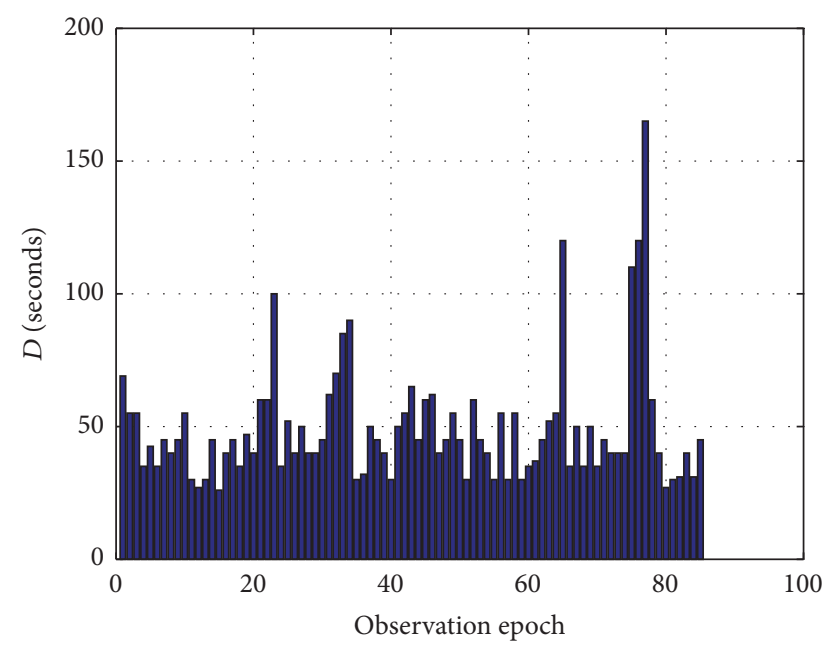

FIGURE 7: Back-to-back duration using period 1 observation data.

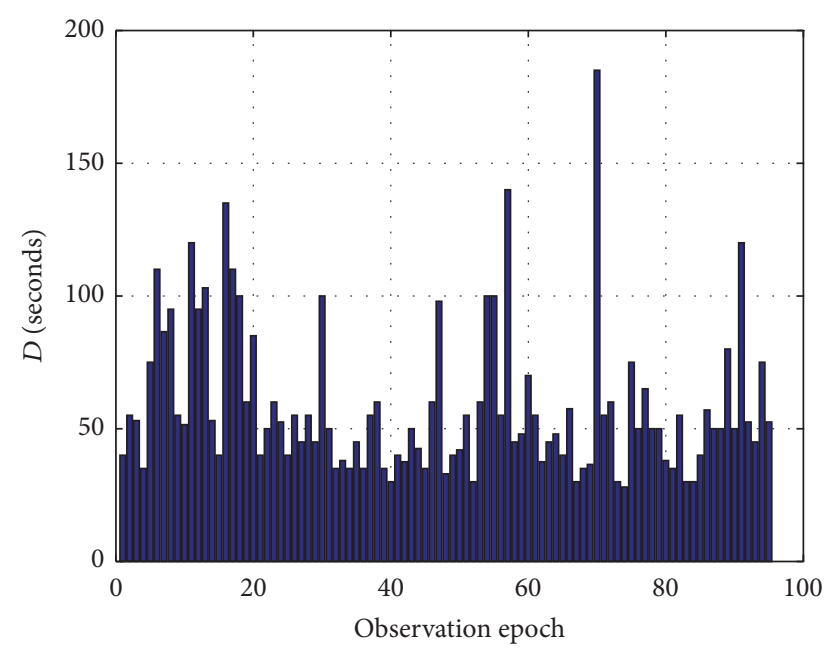

FIGURE 8: Back-to-back duration using period 2 observation data.

$e_{1}=$ PKS J0042-4414, $f_{1}=$ PKS J0059+0006, $g_{1}=\mathrm{PKS}$ J0044-3530, and $h_{1}=3 \mathrm{C} 348$

(2) Source string 2: $a_{2}=$ PKS J0240-2309, $b_{2}=$ PKS J0252$7104, c_{2}=$ PKS J0303-6211, $d_{2}=$ PKS J0309-6058, $e_{2}$ $=\mathrm{PKS} \mathrm{J} 0318+1628, f_{2}=\mathrm{PKS} \mathrm{J} 0323+0534, g_{2}=\mathrm{PKS}$ J0351-2744, $h_{2}=$ PKS J0405-1308, $i_{2}=$ PKS J0409-1757, and $j_{2}=3 \mathrm{C} 123$

(3) Source string 3: $a_{3}=$ PKS J0408-6544, $b_{3}=$ PKS J04206544, $c_{3}=$ PKS J0440-4333, $d_{3}=$ PKS J0442-0017,

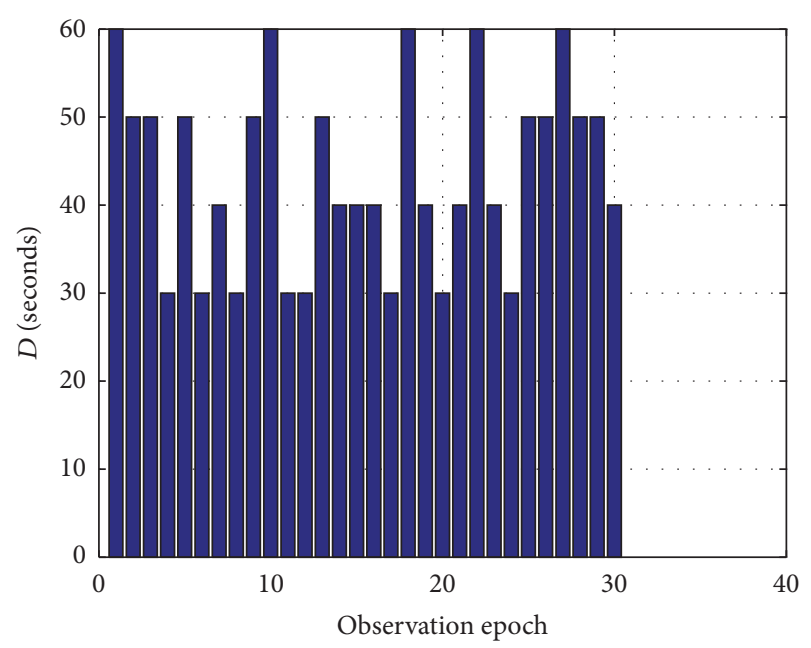

FIGURE 9: Back-to-back duration using period 3 observation data.

$e_{3}=\mathrm{PKS} \mathrm{J0444-2809,} f_{3}=\mathrm{PKS} \mathrm{J0453-2807,} g_{3}=\mathrm{PKS}$ J0519-4546, $h_{3}=$ PKS J0534+1927, $i_{3}=$ PKS J0635$7516, j_{3}=$ PKS J0744-0629, and $k_{3}=$ PKS J0831-1951

Source strings 1, 2, and 3 are observed on 14/11/2012, 28/ $10 / 2012,06 / 11 / 2012$, and 14/11/2012, 06/11/2012 and 14/11/2012, and 14/11/2012 and 06/11/2012, respectively. Assuming that similarity analysis in the intelligent framework takes up to 600 seconds, the achievable increment in the ISL transmit duration is 726 seconds. This increment is applicable to observations conducted on 14/11/2012, 28/10/2012, 06/11/2012, and 14/11/2012, 06/11/2012 and 14/11/2012, and 14/11/2012 and $06 / 11 / 2012$, respectively.

Source substrings $\left(a_{1}-g_{1}\right),\left(a_{1}-e_{1}\right),\left(a_{2}-i_{2}\right)$, and $\left(a_{2}-d_{2}\right)$ repeatedly occur on $16 / 02 / 2012,05 / 02 / 2013,28 / 10 / 2012$, and $06 / 11 / 2012$ and $06 / 11 / 2012$ and 05/02/2013. The transmit durations associated with substrings $\left(a_{1}-g_{1}\right),\left(a_{1}-e_{1}\right),\left(a_{2}-i_{2}\right)$, and $\left(a_{2}-d_{2}\right)$ are 360 seconds, 320 seconds, 340 seconds, and 190 seconds, respectively.

Source substrings $\left(a_{3}-h_{3}\right),\left(i_{3}-k_{3}\right),\left(a_{3}-g_{3}\right)$, and $\left(i_{3}-j_{3}\right)$ repeatedly occur on 28/10/2012 (2 epochs) and 06/11/2012 (2 epochs), respectively. The transmit durations associated with substrings $\left(a_{3}-h_{3}\right),\left(i_{3}-k_{3}\right),\left(a_{3}-g_{3}\right)$, and $\left(i_{3}-j_{3}\right)$ are $360 \mathrm{sec}-$ onds, 160 seconds, 300 seconds, and 120 seconds, respectively. These strings and substrings show that the observations of some astronomy sources are repeated. Therefore, the interference protection framework is feasible. ISLs can exploit these transmit opportunities when they are aware of the astronomy database. In the event that the intelligent framework is 
TABLE 3: Cost figures used to simulate ownership costs.

\begin{tabular}{lccc}
\hline S/N & Component & Cost (USD) & Reference \\
\hline 1 & Conversion of unused earth station & 100,000 & Hoare and Rawlings [10] \\
2 & Internet link from CCE to SVB & 1,000 & \\
3 & Control software per telescope & $20,000(20 \%$ of conversion cost) & Kemball and Cornwell [37] \\
4 & Cyclostationary sensing module & 11,413 & LeMay [38] \\
\hline
\end{tabular}

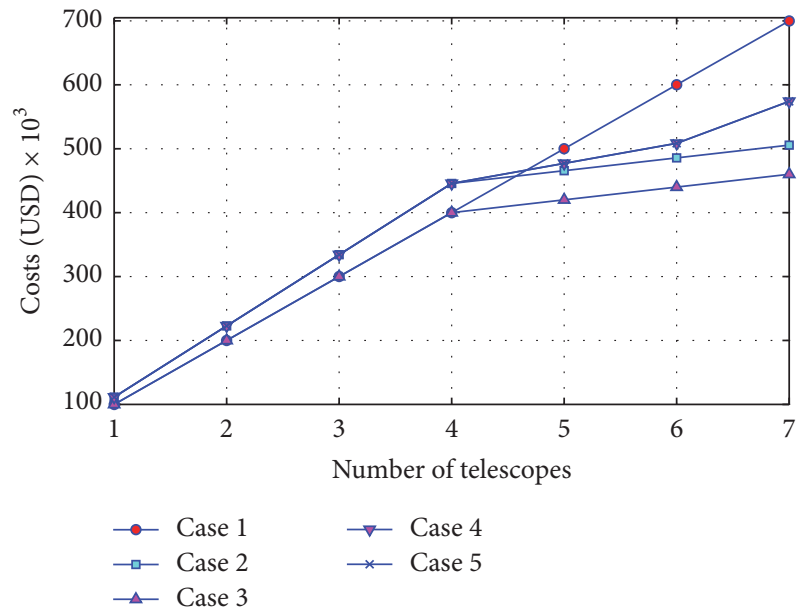

FIGURE 10: Terrestrial radio astronomy organisation ownership costs.

not incorporated, the maximum and minimum transmit durations are 49.5 seconds and 43.7 seconds, respectively. These maximum and minimum values describe the range of the back-to-back connection duration in the absence of the intelligent framework. The range of the average backto-back connection duration is $43.7-49.5$ seconds. When the proposed intelligent framework is used, the range of the average back-to-back connection duration is 769.7-775.5 seconds.

In addition, the costs of ownership and angular resolution of the terrestrial radio astronomy organisation that makes use of primary and secondary GTs are simulated and shown in Figures 10 and 11, respectively. The simulation is conducted for five cases, that is, Cases 1, 2, 3, 4, and 5. The cost of ownership is computed using the parameters given in Table 3.

The cases can be described as follows.

Case 1. The terrestrial radio astronomy organisation has seven primary GTs that are observed in the IEEE UHF band. The primary GTs do not have cyclostationary detectors and are not susceptible to terrestrial wireless network interference. This case describes the kind of scenario found in [11] because it does not use secondary GTs. The baseline is $2100 \mathrm{~km}$.

Case 2. The terrestrial radio astronomy organisation uses four primary GTs and three secondary GTs. The secondary GTs are capable of processing satellite communications packets and astronomy source radio signals. Both primary and secondary GTs operated in the IEEE UHF band. The

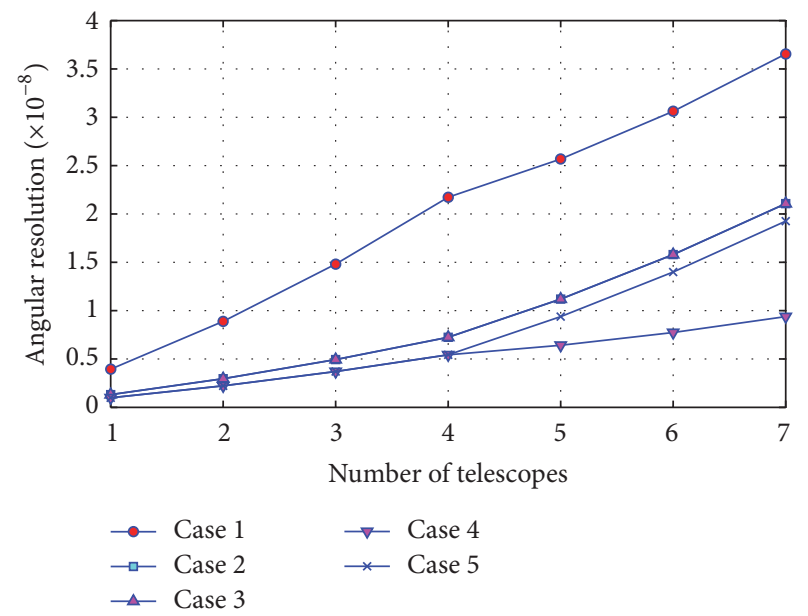

FIGURE 11: Terrestrial radio astronomy organisation angular resolution.

inclusion of secondary GTs doubles the baseline. Primary GTs incorporate a cyclostationary detection mechanism. Secondary GTs are not in terrestrial wireless network vicinity.

Case 3. The terrestrial radio astronomy organisation uses four primary GTs and three secondary GTs operating in the IEEE UHF and IEEE C bands, respectively. Primary GTs do not incorporate a cyclostationary detector for interference protection. Secondary GTs are unaffected by terrestrial wireless network interference. The baseline is $4200 \mathrm{~km}$.

Case 4. The terrestrial radio astronomy organisation uses four primary GTs and three secondary GTs that operate in the IEEE UHF band. The primary and secondary GTs incorporate cyclostationary detectors. The baseline is $4200 \mathrm{~km}$.

Case 5. The terrestrial radio astronomy organisation uses four primary GTs and three secondary GTs that operate in the IEEE UHF band and IEEE $C$ band, respectively. The maximum separating distance between primary and secondary GTs is $4200 \mathrm{~km}$. Both primary and secondary GTs incorporate the cyclostationary detection mechanism.

As shown in Figure 10, the use of secondary GTs alongside primary GTs reduces the terrestrial radio astronomy organisation's ownership costs. In the case where the number of GTs is between five and seven, the cost of ownership is maximum in Case 1 compared to Cases 2, 3, 4, and 5, respectively. When compared to the cost in Case 1, the use of secondary GTs in Cases 2, 3, 4, and 5 reduces ownership costs by $17.9 \%, 25.6 \%$, 
$12.6 \%$, and $12.6 \%$, respectively. The costs in Cases 4 and 5 are equal because these cases are differentiated only by the GT observation frequency. The simulation parameters shown in Table 3 are not observation frequency dependent.

It can also be seen that the cost in Case 1 is lowest when there are up to four primary GTs compared to Cases 2, 3, 4 , and 5 , respectively. This is because the primary GTs in Case 1 do not have any cyclostationary module, multimode control software or Internet link costs. The cyclostationary module increases the cost in Cases 4 and 5. The inclusion of the control software and Internet link increases the cost in Cases 2, 3, 4, and 5. Therefore, the increased cost is due to the incorporation of the features proposed in this paper. The cost of adding the incorporated features increases the cost for the first four GTs for Cases 2, 4, and 5 when compared to Case 1. It does result in an increase for the cost comparison between Cases 1 and 3. This is because, in Cases 1 and 3, the first four GTs are primary GTs with similar functionalities. The average increase in costs of Cases 2, 4, and 5 compared to Case 1 is observed to be the same and equals $11.4 \%$. The increase in cost is equal because only the cyclostationary module is added to the primary GT in Cases 2, 4, and 5 when compared to Case 1.

Further analysis of the results presented in Figure 11 shows that the angular resolution in Cases 2, 3, 4, and 5 outperforms that of Case 1 by $67.5 \%, 59.2 \%, 75 \%$, and $66.7 \%$ on average, respectively. It can be seen that the opportunistic use of secondary GTs enhances the angular resolution. The incorporation of the cyclostationary module in Cases 4 and 5 also enhances the angular resolution because of the interference protection capability.

The improvement in angular resolution is larger when primary and secondary GTs use the IEEE UHF band as seen in Cases 2 and 4 because of the shorter wavelength. Nevertheless, secondary GT incorporation enhances angular resolution. Therefore, using a secondary GT that incorporates the cyclostationary detector improves the angular resolution when its inclusion increases the baseline. Hence, terrestrial radio astronomy organisations should combine primary and secondary GTs to reduce ownership costs and improve angular resolution.

5.2. Opportunistic Computing Scheme (OCS). The probability of success of the opportunistic computing scheme (OCSSEP) is also investigated. OCS-SEP is dependent on the number of GTs and HPC computational units (CUs). The OCS-SEP is investigated for different number of GTs and HPC CUs. The simulation result is shown in Figure 12. An increase in the $\mathrm{CU}$ from $10 \mathrm{Kbits}$ to $100 \mathrm{Kbits}$ improves the OCS-SEP for the same number of GTs. An increase in CUs from 10 Kbits to 100 Kbits improves the OCS-SEP from 0.0387 to 0.9087 . The OCS-SEP also improves when the CU increases from $100 \mathrm{Kbits}$ to $1000 \mathrm{Kbits}$. An increase in the CU from $100 \mathrm{Kbits}$ to $1000 \mathrm{Kbits}$ increases the OCS-SEP from 0.0726 to 0.9085 . Hence, the availability of more HPC CUs improves the OCS-SEP.

The influence of OCS on terrestrial wireless network throughput is also investigated. The terrestrial wireless network throughput is that obtained when OCS execution is

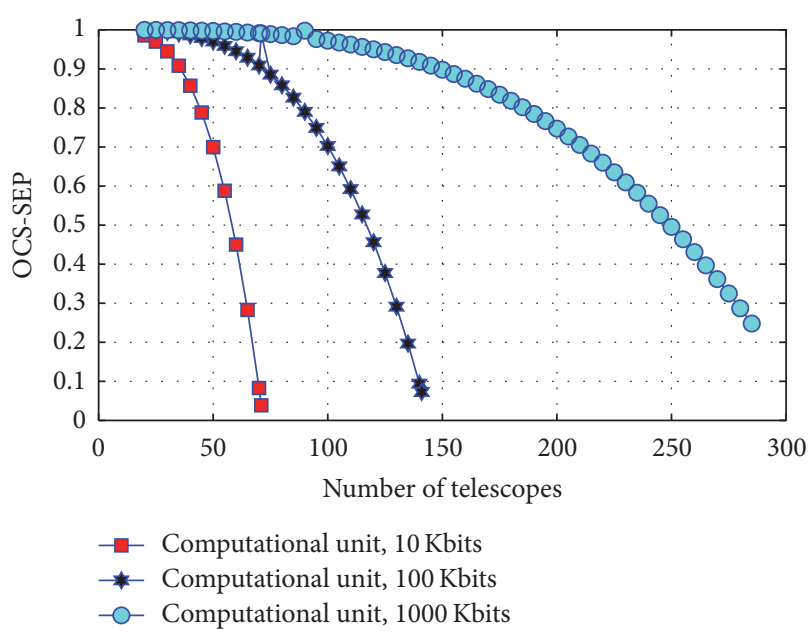

FIGURE 12: OCS-SEP for varying computational units and number of telescopes.

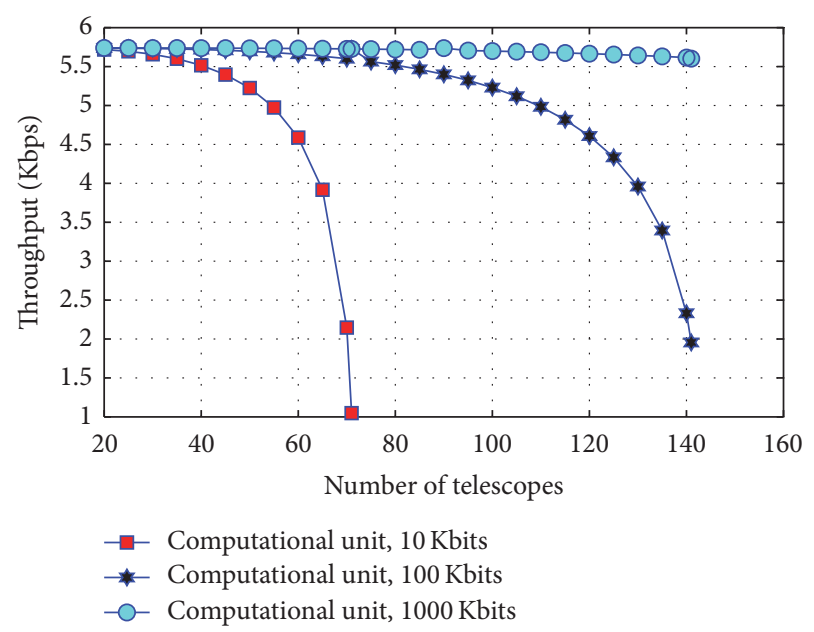

FIGURE 13: Relationship between terrestrial wireless network throughput and number of telescopes.

used to develop artificial neural network multiuser detectors. The simulation environment considers a scenario where three CRs share a channel to improve spectrum utilisation. The simulated OCS throughput is shown in Figure 13.

As seen in Figure 13, the throughput reduces with a decreasing HPC CU. The lowest throughput is obtained at epochs where available CUs cannot support the number of functional GTs. The achievable throughput is minimum when the HPC has 10 Kbits and there are 71 GTs and when the HPC has 100 Kbits and there are 141 GTs.

Further analysis shows that the CBS throughput is enhanced by $60.4 \%$ on average when the HPC CU is $1000 \mathrm{Kbits}$ compared to when the HPC CU is 10 Kbits for up to 71 telescopes. The CBS throughput is enhanced by $59.3 \%$ on average when the HPC has 100 Kbits compared to when the HPC has $10 \mathrm{Kbits}$ with up to $71 \mathrm{GTs}$. When there are up to 141 GTs, the increase in HPC CU from 100 Kbits to 1000 Kbits improves throughput by an average of $37.7 \%$. 


\section{Conclusion}

This paper addresses challenges affecting the future conduct of terrestrial radio astronomy observations. The terrestrial radio astronomy organisations being considered use ground telescopes realised by converting unused earth stations. The challenges are those of optimising terrestrial radio astronomy observations and enhancing high performance computing infrastructure utilisation. The optimisation goals aim to protect terrestrial radio astronomy observations from intersatellite interference and enhance angular resolution. The interference mitigation framework utilises the similarities in the order of observed astronomy sources. The use of similarities protects ground telescopes from intersatellite link interference and increases intersatellite link connection duration. The paper also proposes the use of secondary telescopes to enhance angular resolution. In addition, the paper proposes the opportunistic computing scheme to enhance high performance computing infrastructure utilisation. The opportunistic computing scheme is a synergy between radio astronomy observations and cognitive base stations. It enhances cognitive base station autonomy. Investigations show that the intersatellite links that use cognitive radios with the proposed intelligent framework have an interference-free connection duration lying between 43.7 seconds and 49.5 seconds. The interference-free intersatellite link transmission duration is increased when the similarity in radio astronomy observation patterns is considered. Analysis also shows that the opportunistic computing scheme enables the realisation of cognitive base stations. In addition, the opportunistic computing scheme enhances terrestrial wireless network throughput. It is also shown that the use of secondary telescopes enhances angular resolution by up to $59 \%$ and reduces costs by up to $12.6 \%$.

\section{Competing Interests}

The authors declare that they have no competing interests.

\section{Acknowledgments}

The authors would like to thank the researchers at the Communications Research Group, Department of Electrical Engineering, University of Cape Town, for their useful comments. The authors acknowledge the financial support of the National Research Foundation, South Africa (NRF SA), Telkom South Africa, Jasco/TeleSciences, and the Department of Trade and Industry/Technology and Human Resources Programme (DTI/THRIP).

\section{References}

[1] F. Combes, "The square kilometer array: cosmology, pulsars and other physics with the SKA," in Proceedings of the 2nd International Summer School on Intelligent Signal Processing for Frontier Research and Industry, pp. 1-16, Paris, France, July 2014.

[2] R. Deane, Z. Paragi, M. Jarvis et al., "Multiple supermassive black hole systems: SKA's future leading role," Proceedings of Science, Advancing Astrophysics with Square Kilometre Array, pp. 1-11, https://arxiv.org/abs/1501.01238.
[3] T. E. Bell, "Waiting for gravity: gravitational waves would give astronomers an unprecedented view into acts of astronomical violence," IEEE Spectrum, vol. 43, no. 7, pp. 40-46, 2006.

[4] T. Pultarova, "Telecommunications-Space Tycoons go head to head over mega satellite network," Engineering and Technology, vol. 10, no. 2, p. 20, 2015.

[5] M. DePasquade and J. Bradford, Space Works: Nano/Micro Satellite Market Assessment, Revision C, 2013.

[6] M. Poblet, "Affordable telecommunications: a new digital economy is calling," Australian Journal of Telecommunications and the Digital Economy, vol. 1, no. 1, article 12, 2013.

[7] http://www.nanosats.eu/.

[8] A. A. Periola and O. E. Falowo, "Interference protection of radio astronomy services using cognitive radio spectrum sharing models," in Proceedings of the European Conference on Networks and Communications, pp. 301-305, Paris, France, July 2015.

[9] H. R. Klockner, S. Rawlings, I. Heywood et al., "Goonhilly: a new site for e-Merlin and the EVN," in Proceedings of the European VLBI Network Symposium and EVN Users Meeting: VLBI and the New Generation of Radio Arrays, pp. 1-10, Manchester, UK, September 2010, http://www.jive.nl.

[10] M. Hoare and S. Rawlings, "Recycling for radio astronomy," Astronomy and Geophysics, vol. 53, no. 1, pp. 1.19-1.21, 2012.

[11] D. Barbosa, M. Bergano, V. A. R. M. Ribeiro et al., "Design, environmental and sustainability constraints of new African observatories: the example of the Mozambique Radio Astronomy Observatory," in Proceedings of the AFRICON, pp. 1-5, Pointe-Aux-Piments, Mauritius, September 2013.

[12] M. J. Gaylard, M. F. Bietenholz, L. Combrinck et al., "An African VLBI network of radio telescopes," http://arxiv.org/abs/ 1405.7214.

[13] B. D. Asabere, M. J. Gaylard, C. Horellou, H. Winkler, and T. Jarrett, "Radio Astronomy in Africa: the case of Ghana," pp. 16, 2015, http://arxiv.org/pdf/1503.08850.pdf.

[14] L. Woodburn, T. Natusch, S. Weston et al., "Conversion of a New Zealand 30-metre telecommunications antenna into a radio telescope," Publications of the Astronomical Society of Australia, vol. 32, article e017, 14 pages, 2015.

[15] D. Barbosa, A. J. Boonstra, R. Aguiar, A. V. Ardene, De. S. Vela, and L. V. Montnegro, "A Sustainable approach to large ICT Science based infrastructures; the case for Radio Astronomy," in Proceedings of the IEEE International Energy Conference (ENERGYCON '14), pp. 668-674, Cavtat, Croatia, May 2014.

[16] M. N. Derahman, K. Dimyati, A. M. Mohammadi, and M. K. Abdullah, "Improvement of decision making protocol for Duty Cycle Division Multiplexing (DCDM) system," in Proceedings of the 2nd International Conference on Future Networks (ICFN '10), pp. 155-158, Sanya, China, January 2010.

[17] A. Malekmohammadi, M. K. Abdullah, A. F. Abas, G. A. Mahdiraji, and M. Mokhtar, "Absolute Polar Duty Cycle Division Multiplexing (APDCDM); technique for wireless communications," in Proceedings of the International Conference on Computer and Communication Engineering (ICCCE '08), pp. 617-620, Kuala Lumpur, Malaysia, May 2008.

[18] K. Rele, D. Roberson, B. Zhang et al., "A two-tiered cognitive radio system for interference identification in $2.4 \mathrm{GHz}$ ISM band," in Proceedings of the 7th IEEE Consumer Communications and Networking Conference (CCNC '10), pp. 1-5, IEEE, Las Vegas, Nev, USA, January 2010.

[19] P. Woudt, R. P. Fender, R. Armstrong, and C. Carignan, "Early science with the Karoo Array Telescope test array KAT-7," South African Journal of Science, vol. 109, no. 7-8, 2 pages, 2013. 
[20] V. Pankonin and R. M. Price, "Radio astronomy and spectrum management: the impact of WARC-78," IEEE Transactions on Communications, vol. 29, no. 8, pp. 1228-1237, 1941.

[21] P. J. Waterman, "Conducting radio astronomy in the EMC environment," IEEE Transactions on Electromagnetic Compatibility, vol. 26, no. 1, pp. 29-33, 1984.

[22] D. R. DeBoer, S. L. C. Pol, M. M. Davis et al., "Radio frequencies: policy and management," IEEE Transactions on Geoscience and Remote Sensing, vol. 51, no. 10, pp. 4897-4907, 2013.

[23] T. E. Gergely, "Spectrum access for the passive services: the past and the future," Proceedings of the IEEE, vol. 102, no. 3, pp. 393398, 2014.

[24] T. E. Gergely, "The SKA, RFI and ITU regulations," in Proceedings of the Workshop on Mitigation of Radio Frequency Interference in Radio Astronomy, pp. 1-10, Penticton, Canada, July 2004.

[25] I. Kucuk, "Radio astronomy in turkey: site selection studies for radio quiet zones," in Proceedings of the RFI Mitigation Workshop, Groningen, The Netherlands, March 2010.

[26] R. Umar, Z. Z. Abidin, Z. A. Ibrahim, Z. Rosli, and N. Noorazlan, "Selection of radio astronomical observation sites and its dependence on human generated RFI," Research in Astronomy and Astrophysics, vol. 14, no. 2, pp. 241-248, 2014.

[27] R. Umar, Z. Z. Abidin, and Z. A. Ibrahim, "The importance of site selection for radio astronomy," Journal of Physics: Conference Series, vol. 539, no. 1, Article ID 012009, 2014.

[28] N. H. Sabri, R. Umar, W. Z. A. Wan Mokhtar et al., "Preliminary study of vehicular traffic effect on radio signal for radio," Jurnal Teknologi, vol. 75, no. 1, pp. 313-318, 2015.

[29] M. J. Bentum, A. J. Boonstra, and W. A. Baan, "Impact of cognitive radio on radio astronomy," in Proceedings of the RFI Mitigation Workshop, pp. 1-7, Groningen, The Netherlands, March 2010.

[30] J. M. Ford and K. D. Buch, "Mitigation techniques in radio astronomy," in Proceedings of the IEEE International Geoscience and Remote Sensing Symposium, pp. 231-234, Québec, Canada, July 2014

[31] H. Asadi, H. Volos, M. M. Marefat, and T. Bose, "Metacognition and the next generation of cognitive radio engines," IEEE Communications Magazine, vol. 54, no. 1, pp. 76-82, 2016.

[32] T. V. D. Zant, M. Kouw, and L. Schomaker, "Generative artificial intelligence," in Philosophy and Theory of Artificial Intelligence, V. C. Muller, Ed., vol. 5, pp. 103-120, 2012.

[33] K. Tsakgaris, A. Bantouna, and P. Demestichas, "Self-organizing maps for advanced learning in cognitive radio systems," Computers and Electrical Engineering, vol. 38, no. 4, pp. 852-870, 2012.

[34] K. P. Bagadi and S. Das, "Multiuser detection in SDMA-OFDM wireless communication system using complex multilayer perceptron neural network," Wireless Personal Communications, vol. 77, no. 1, pp. 21-39, 2014.

[35] P. Andreani, ALMA Cycle 3 Proposer's Guide and Capabilities, Doc 3.2, Ver 1.9, 2015.

[36] S. J. Almalki and J. Yuan, "A new modified Weibull distribution," Reliability Engineering and System Safety, vol. 111, pp. 164-170, 2013.

[37] A. J. Kemball and T. J. Cornwell, "A simple model of software costs for the square kilometre array," Experimental Astronomy, vol. 17, no. 1-3, pp. 317-327, 2004.

[38] B. LeMay, Agilent Technologies Introduces Basic Spectrum Analyzer for Budget-Driven Applications, http://www.agilent .com/about/newsroom/presrel/2012/30nov-em12148.html. 

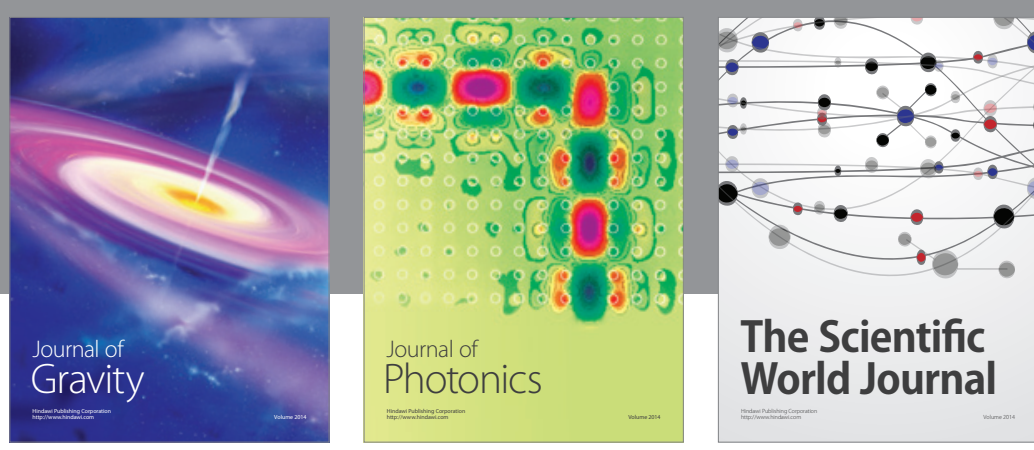

The Scientific World Journal
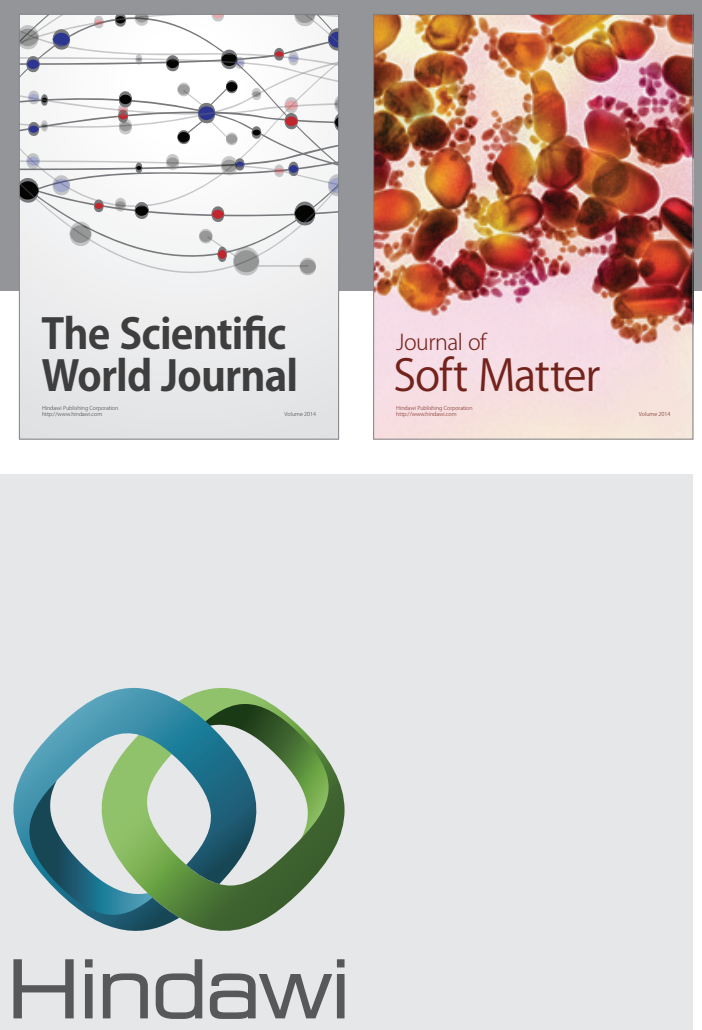

Submit your manuscripts at

http://www.hindawi.com

nternational Journal of

Statistical Mechanics
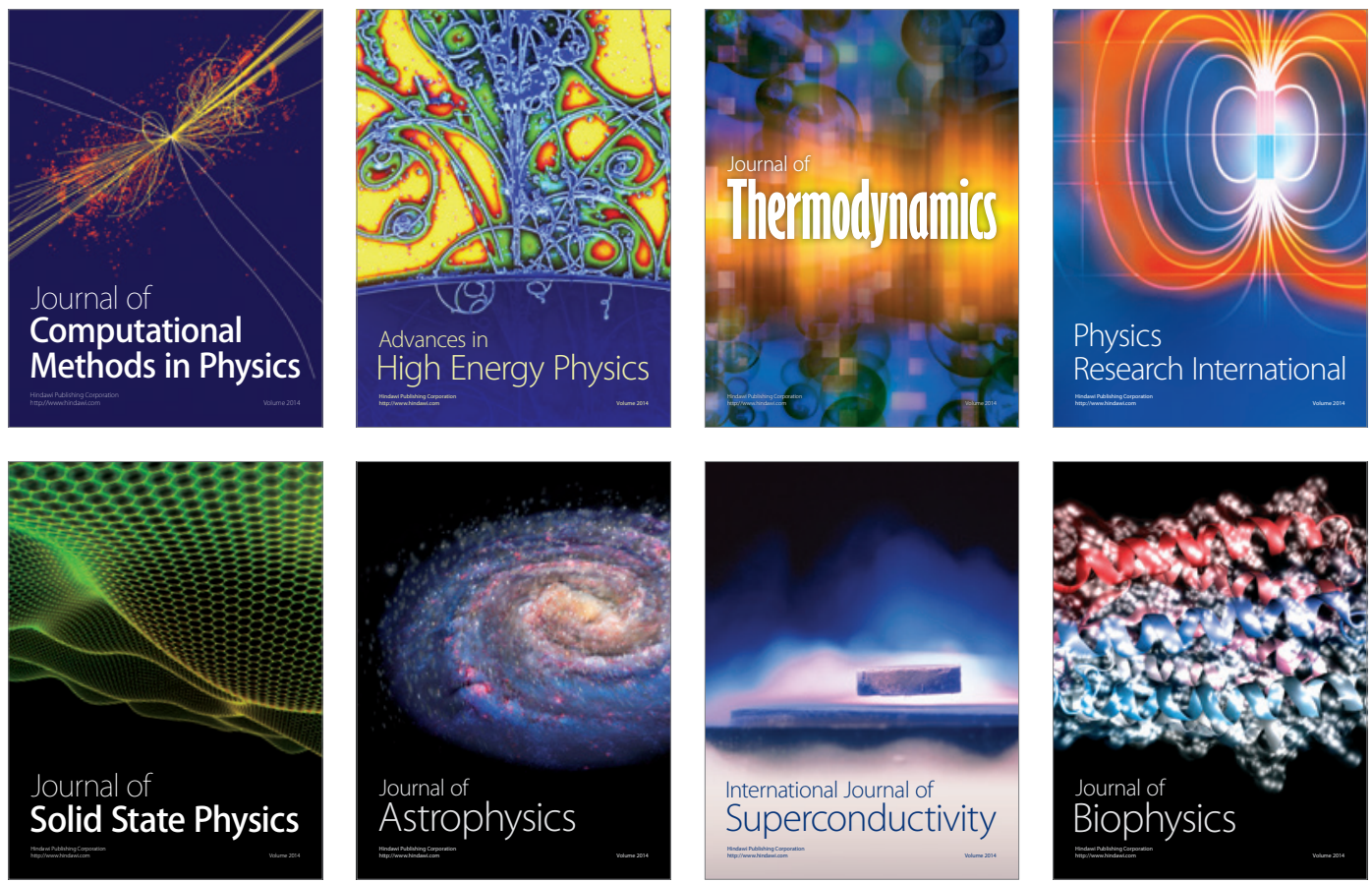
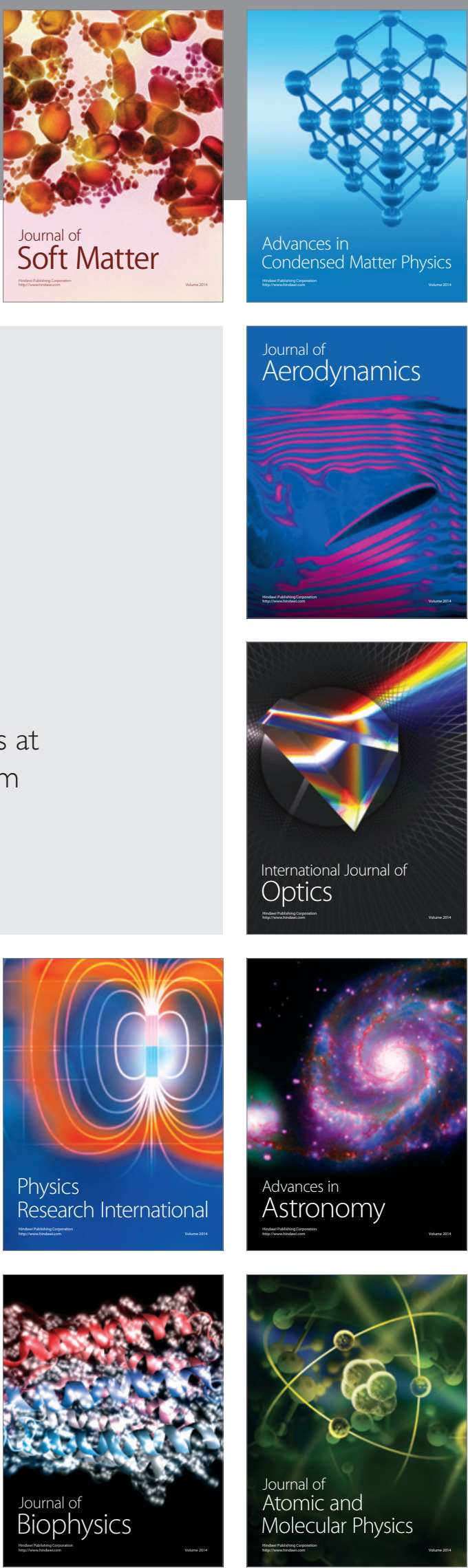